\title{
The PLC control and the monitoring configuration software monitoring design of sewage treatment system
}

\author{
Bai Yan ${ }^{1,}$, Zheng Wengang ${ }^{2}$, Zhou Jingbo ${ }^{3, c}$ \\ ${ }^{1}$ BeiHua University Engineering Training Center \\ ${ }^{2}$ Beihua University Mechanical Engineering College \\ ${ }^{3}$ Funing Qinhuangdao district power supply company \\ abyw1689@sohu.com, ${ }^{b} 745575912 @ q q . c o m$
}

Keywords: sewage treatment; PLC; process flow

\begin{abstract}
With the global water crisis is getting more and more serious, industrial and sewage treatment are becoming the urgent problem need to be solved.In this paper, sewage treatment project of a factory is research object, the design using the upper computer configuration software from YAKONG of China which named KINGVIEW6.53 and using the PLC from Siemens Ltd. The automatic control of PLC has been successfully completed in sewage treatment ,which has improved the operation efficiency and management level of sewage treatment equipment.
\end{abstract}

\section{Introduction}

With the society progressing, the economic development, and the unceasing improvement of living standard, human can reform the ecological environment more effectively and largely. The sewage treatment industry has taken up an important position. But compared it in other advanced countries, our sewage treatment industry falls behind comparatively [1].Therefore, studying on the automatic sewage treatment which is based on the technology of computer control has important practical significance.

This essay introduces the main process flow of sewage treatment. According to the handicraft characteristic and the reaction quest of the sewage treatment, the essay will not only raises the systematic software design plan of the sewage treatment which is for S7-300PLC and kingview6.53, but also carries out the whole introduction and the entire designing plan about the software function getting off place machine on navar.

\section{The overall design of automatic control system of sewage treatment}

Sewage treatment automatic control system is divided into two levels of upper and lower computers, YanHua IPC is selected as monitoring management computer, SIEMENSS7-300PLC is selected as lower computer to control field,In view of the complexity of sewage treatment, the control function and configuration of each PLC control station is introduced in details, and the configuration screen of monitoring management is designed too.

\section{Sewage treatment process.}

Sewage treatment process as shown in Fig.1.

Sewage from inlet system through the coarse grid and cleaning machine is preliminarily eliminated the bulk impurity objects, where to remove sand pool at. In addition to the sand tank system fine grille and basic cleaning machine further purification of fine particles in the sewage objects, the small sand filter in sewage in oxidation ditch reactor. In addition to the sand tank system fine grille and basic cleaning machine further purification of fine particles in the sewage objects, the small sand filter in sewage in oxidation ditch reactor-In addition to oil, disinfection, adjust the $\mathrm{pH}$ value of sewage, The content of dissolved oxygen in sewage will change through the detection of the oxygen content in the sewage, feedback to the PLC control aerator converter operation [2]. The submersible mixer is the role of the water, the sewage and sludge oxidation ditch in the intense mixing make contact, the biochemical reaction is fuller. To maximize the 
decomposition of harmful components in sewage treated sewage into the sedimentation tank, sewage treatment by sedimentation tank to reach the final link of clean water dehydration, dehydration process under the action of centrifugal dewatering machine.

\section{Automatic control of sewage treatment system.}

Control the way "concentration monitor management, scatter a control" of the system adoption. The emulation hold, place of honor machine, gather a line machine, printer to be located on medium control room, two set PLC are located on control the spot. Adopt this kind of structure can make the information in the production line which can centralize management with carry out the whole operation, management with excellent turns. Control dangerous dispersion, in the meantime raising the system credibility also.

Recently, for carrying out high quality, low consume stable dependable movements. Many domestic and international waste water treatment factories adopted a modern forerunner to control technique, calculator technique and constitute calculator direct control.

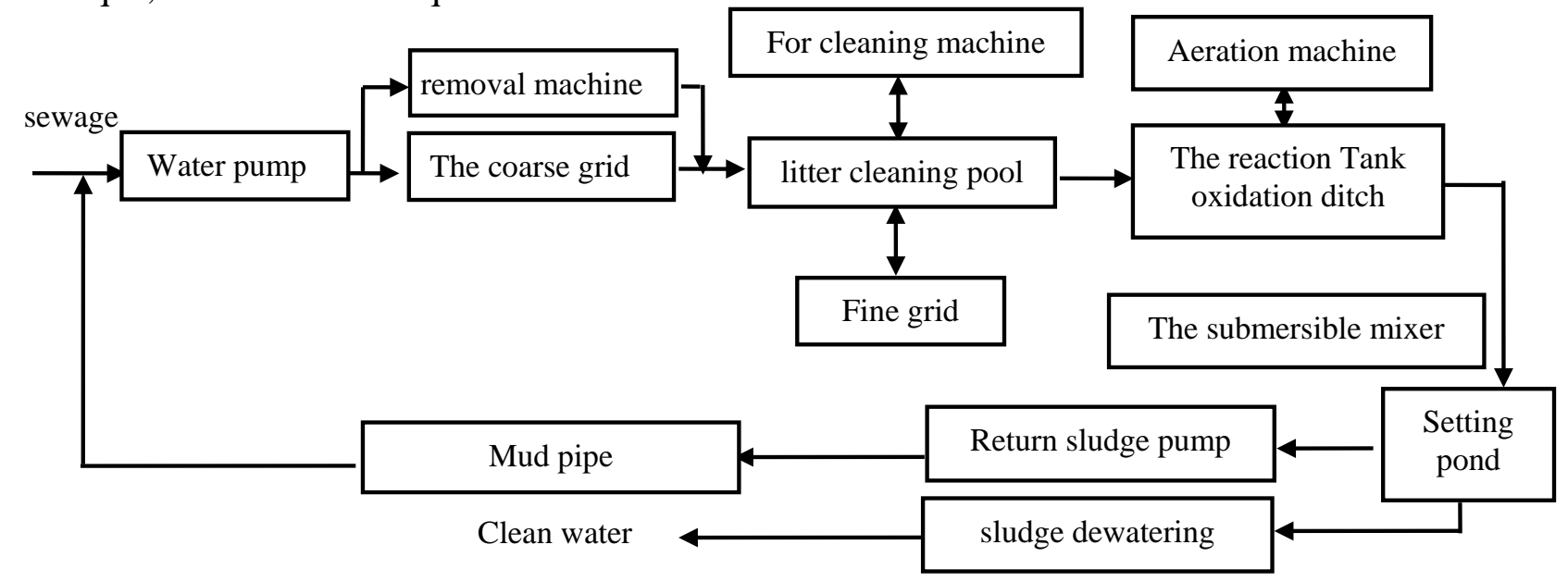

Fig.1. Sewage treatment process

PLC as the control system of industrial sewage treatment system makes the design process simpler, can realize the function becomes more efficient [3].The part of the PLC wiring diagram as shown in Fig. 2

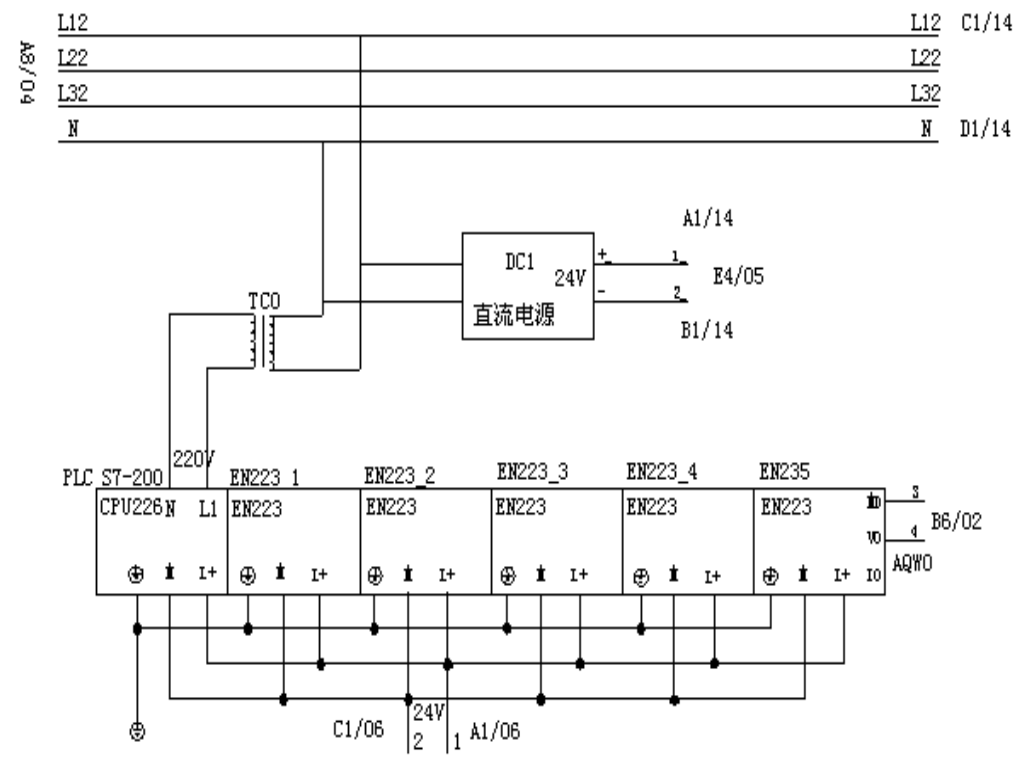

Fig.2.The part of the PLC wiring diagram

\section{Process control of aeration system.}

The aeration system, for example, control of aeration system program mainly about two sets of the aerator operations: speed and stop. The working process includes the following aspects. 
Start 1\# the aerator, the frequency operation. Detection of oxygen content and frequency converter speed, if the oxygen content is lower than the setting value and the velocity is greater than or equal to $100 \%$, then $1 \#$ the aerator variable frequency to frequency operation, $2 \#$ the aerator variable frequency operation, If the oxygen content is lower than the setting value and the velocity is greater than or equal to $100 \%$, then $2 \#$ the aerator power frequency operation. If the oxygen content is greater than the set value, the removal of the aerator operating frequency $2 \#$, and start the aerator operating frequency $1 \#$.

\section{The configuration design of wastewater treatment system}

\section{The software system design.}

Medium control two calculator adoption PII class works of room control machine, and circulate the operate system of Windows NT. The place of honor machine is responsible for on the scene establish each long range control (full-automatic control, automatic control, order to move a control) with surveillance, make use of Intouch7. 0 engineering software packages provide a good person machine dialogue boundary but obtain concerning parameters and equipments appearance information from the PLC by searching a way. To keep a view manifestation to come out on the CRT by good sketch type, print a necessary data in the meantime of being provided for lord to control and manage instruction produce. Supervise and control boundary but include the system total diagram, system cent diagram, imitating the quantity general chart, parameter a constitution, report to the police a window, history trend and register a window etc. boundary but. In the system total diagram, circulate the personnel can keep watching on signals, such as all movement appearances [5], equipments trouble and liquid which control an equipments and discharge...etc. Operate a personnel in the system the cent the diagram can carry on a single machine point to move to circulate an operation to all control equipments, and then become the set automatically circulate operation or the whole factories full-automatic operation combine surveillance. In the meantime place of honor machine 2 and does a plait distance a machine. In order to keep the value of craft process of the view manifestation whole factory and main parameter, medium control room to establish another inset type emulation to hold, has been one control by the place of honor machine, The software system structure diagram as shown in Fig.3.

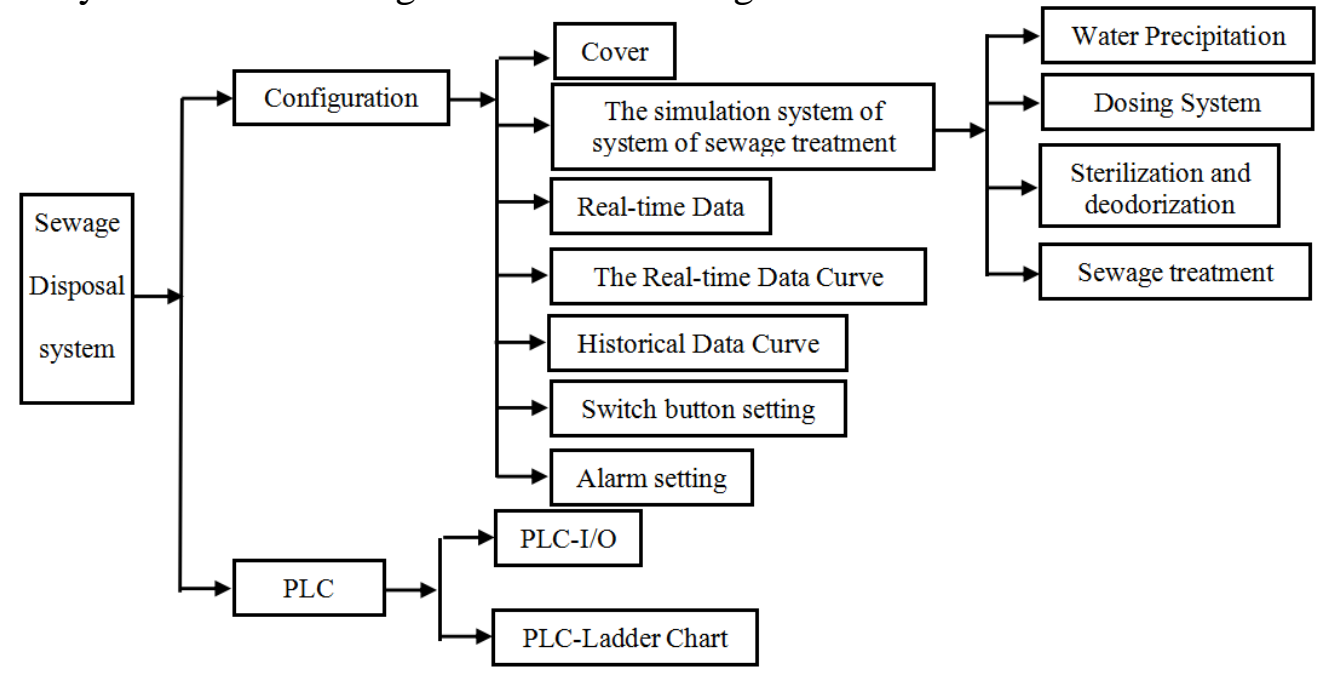

Fig.3.The software system structure diagram

\section{Configuration interface design.}

The configuration screen of monitoring management as shown in Fig.4.

\section{The dynamic interface program design.}

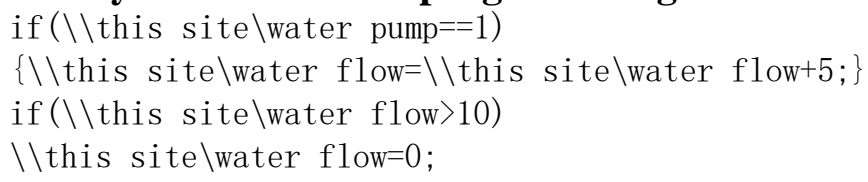


if ( $\backslash$ this site $\backslash$ sump pump==1)

$\backslash \backslash$ this site $\backslash$ sump pump $=\backslash \backslash$ this site $\backslash$ sump pump $+5 ;\}$

if ( \\this site \sump pump $>10)$

$\backslash \backslash$ this site $\backslash$ sump pump $=0$;

if $(\backslash \backslash$ this site $\backslash$ sludge pump==1)

$\{\backslash \backslash$ this site $\backslash$ sludge pump $==\backslash \backslash$ this site $\backslash$ sludge pump $=+5 ;\}$

if ( $\backslash \backslash$ this site $\backslash$ sludge pump $=>10)$

$\backslash \backslash$ this site $\backslash$ sludge pump $==0$;

Monitoring software to display some data curve shown in Fig.5.

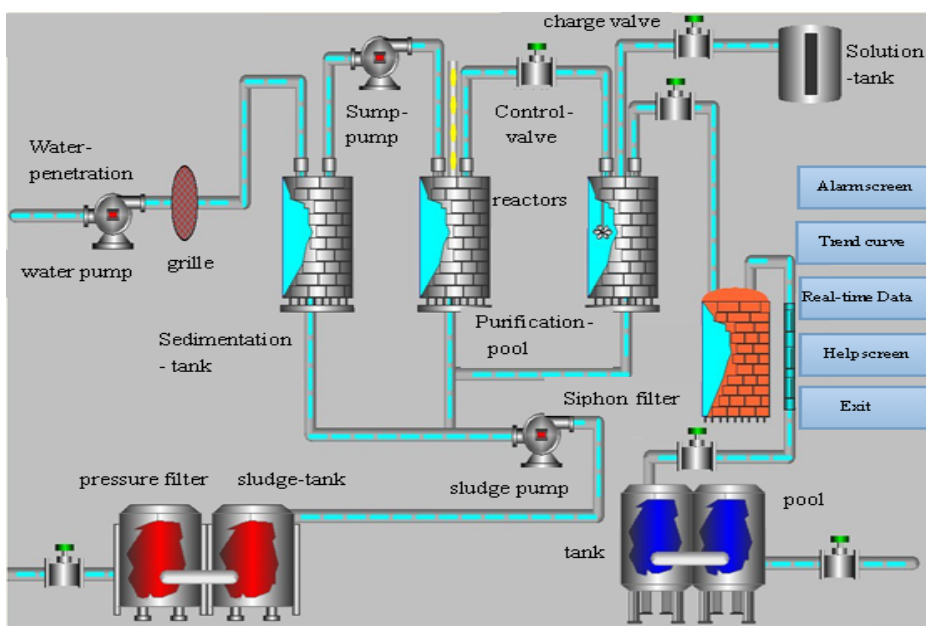

Fig.4. the configuration screen of monitoring management

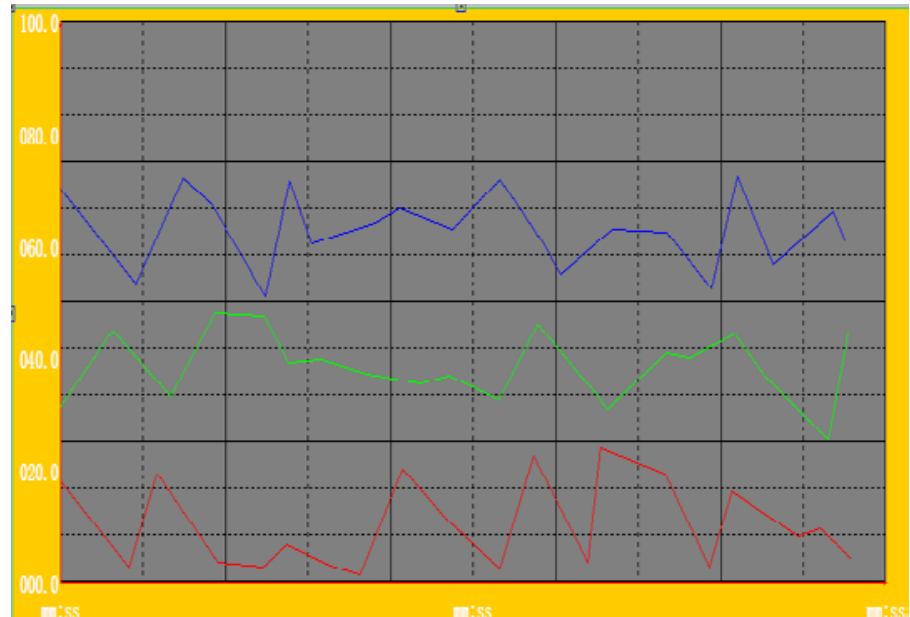

Fig.5. the aeration system of curves

\section{Conclusion}

Industrial automatic control system of sewage treatment plant design, installation is completed.The system debugging and trial operation is determining compliance with each control parameter of the actual water quantity and water quality, to find and solve the equipment, instruments, procedures, process and other aspects of the problems, inspection system. It is the realization process design goal, namely the effluent can reach the design indexes requirements.

\section{References}

[1] Wan T J.An application of artificial neuromolecular system for effluent quality prediction of wastewater treatment plant. Chinese Instrument Environment Engineering. 2000

[2] Jiang Wei. System Identification Methods and Applications. National Defence Industry Press. 2010 
[3] M Batty.The AJAX project:New theory,and new software for space syntax[J].SPACE SYNTAX 5TX INTERNATIONAL SYMPOSIUM 2005,VOL 1,PROCEEDINGS.2005,(12/1):10 11

[4] Chen J C,Chang N B.Assessing wastewater reclamation potential by neural network model. Engineering Applications of Artificial Intelligence. 2003

[5] Du Y G,Tyagi R D,Bhamidimarri R.Use of fuzzy neural-net model for rule generation of activated sludge process. Process Biochemistry. 1999 